\title{
Nonlinear dynamics for local fractional Burgers' equation arising in fractal flow
}

\author{
Xiao-Jun Yang • J. A. Tenreiro Machado • \\ Jordan Hristov
}

\begin{abstract}
The local fractional Burgers' equation (LFBE) is investigated from the point of view of local fractional conservation laws envisaging a non-linear local fractional transport equation with a lin-ear non-differentiable diffusion term. The local frac-tional derivative transformations and the LFBE conver-sion to a linear local fractional diffusion equation are analyzed.
\end{abstract}

Keywords Conservation laws · Burgers' equation - Transport equation - Diffusion equation · Local fractional derivative

\section{Introduction}

The Burgers' equation (BE) [1-3] is the simplest non-linear diffusion equation arising in the fluid mechanics. The BE can be transformed into the diffusion equation by means of the Hopf-Cole transformation as shown in [4,5]. On the other hand, the conservation laws for BE were discussed in [6]. The BE was analyzed in a broad perspective, namely with singular data [7], for a non-commutative form [8], in lattice gas problems [9], and by means of a stochastic approach [10]. The BE has been successfully applied to turbulence prob-lems [11], traffic flow [12] and plane waves [13]. The numerical solution of the BE was developed by finite element method [14], generalized boundary element method [15], tanh-coth method [16] and other methods (see also cited references therein).

In view of the fractional calculus theory [17-21], applicable to nonlinear problems on science and engi-neering, the adoption of fractional BEs was suggested [22] and several solutions were developed $[23,24]$ and analyzed [25,26]. The solution strategies employed homotopy analysis [27] and Adomian decomposition methods [28] to solve the space- and time-fractional versions of the fractional BE. In this context, the clas-sic finite difference method was proposed to solve the generalized FBE [29]. Furthermore, the variational iter-ation method (VIM) was successfully applied for tak-ing the Burgers' flows with fractional derivatives [30]. The coupled BEs within time- and space-fractional derivatives were solved by the Adomian decomposition 
method in [31]. We can mention also the generalized differential transformation and homotopy perturbation methods that were adopted to solve the time-fractional BEs [32].

Recently, the local fractional calculus was successfully applied to non-differentiable problems arising in the areas of solid mechanics [33], heat transfer and wave propagation [34], diffusion [35], hydrodynamics [36], vehicular traffic flow [37] and other topics [3842] (see also references therein).

The present manuscript focuses on the LFBE arising from the nonlinear local fractional transport equation involving a linear non-differentiable diffusion term with the local fractional conservation laws. This article is structured as it follows. In Sect. 2, the nonlinear local fractional transport equation from the local fractional conservation laws is introduced. In Sect. 3, the LFBE arising in fractal flow is discussed. In Sect. 4, the local fractional derivative transformation is suggested. In Sect. 5, the results are discussed. Finally, Sect. 6 outlines the main conclusions.

\section{The nonlinear local fractional transport equation via local fractional conservation laws}

Let us consider a nonlinear local fractional transport equation from the local fractional conservation laws point of view. In this context, the local fractional partial derivative of the non-differentiable function $f(x, y)$ with respect to $x=x_{0}(0<\alpha<1)$ is defined as $[33,35]$ :

$$
\frac{\partial^{\alpha} f\left(x_{0}, y\right)}{\partial x^{\alpha}}=\lim _{x \rightarrow x_{0}} \frac{\Delta^{\alpha}\left(f(x, y)-f\left(x_{0}, y\right)\right)}{\left(x-x_{0}\right)^{\alpha}},
$$

where

$$
\begin{aligned}
& \Delta^{\alpha}\left(f(x, y)-f\left(x_{0}, y\right)\right) \\
& \cong \Gamma(1+\alpha)\left[f(x, y)-f\left(x_{0}, y\right)\right] .
\end{aligned}
$$

If $Q(x, t)$ denotes a fractal flow and its conserved density is $\phi(x, t)$, then we have [37]

$$
\begin{aligned}
& Q(t)=\frac{1}{\Gamma(1+\alpha)} \int_{x_{1}}^{x_{2}} \frac{\partial^{\alpha} \phi(x, t)}{\partial t^{\alpha}}(\mathrm{dx})^{\alpha}, \\
& Q(t)=\frac{1}{\Gamma(1+\alpha)} \int_{x_{1}}^{x_{2}} \frac{\partial^{\alpha} Q(x, t)}{\partial x^{\alpha}}(\mathrm{dx})^{\alpha} .
\end{aligned}
$$

The local fractional integral operator of $f(x)$ of order $\alpha$ in the interval $[a, b]$ is defined as $[33,35]$

$$
\begin{aligned}
{ }_{a} I_{b}^{(\alpha)} f(x) & =\frac{1}{\Gamma(1+\alpha)} \int_{a}^{b} f(t)(d t)^{\alpha} \\
& =\frac{1}{\Gamma(1+\alpha)} \lim _{\Delta t \rightarrow 0} \sum_{j=0}^{j=N-1} f\left(t_{j}\right)\left(\Delta t_{j}\right)^{\alpha},
\end{aligned}
$$

with the partitions of the interval $[a, b],\left(t_{j}, t_{j+1}\right), j=$ $0, \ldots, N-1, t_{0}=a$ and $t_{N}=b$, for $\Delta t_{j}=t_{j+1}-t_{j}$ and $\Delta t=\max \left\{\Delta t_{0}, \Delta t_{1}, \Delta t_{j}, \ldots\right\}$.

Employing expressions (3) and (4), we have [37]:

$$
\frac{1}{\Gamma(1+\alpha)} \int_{x_{1}}^{x_{2}}\left\{\frac{\partial^{\alpha} \phi(x, t)}{\partial t^{\alpha}}+\frac{\partial^{\alpha} Q(x, t)}{\partial x^{\alpha}}\right\}(\mathrm{dx})^{\alpha}=0 .
$$

Hence, the local fractional conservation law reads as [37]

$\frac{\partial^{\alpha} \phi(x, t)}{\partial t^{\alpha}}+\frac{\partial^{\alpha} Q(x, t)}{\partial x^{\alpha}}=0$,

where the functions $\phi(x, t)$ and $Q(x, t)$ are the conserved density and fractal flow, respectively.

If the fractal flow can be represented as

$Q=\frac{\phi^{2}}{2}$,

then the nonlinear transport equation in the local fractional conservation law becomes

$\frac{\partial^{\alpha} \phi}{\partial t^{\alpha}}+\frac{\partial^{\alpha}}{\partial x^{\alpha}}\left(\frac{\phi^{2}}{2}\right)=0$.

Therefore, the nonlinear local fractional transport equation (also known as the local fractional inviscid BE) takes the form

$\frac{\partial^{\alpha} \phi}{\partial t^{\alpha}}+\phi \frac{\partial^{\alpha} \phi}{\partial x^{\alpha}}=0$.

The linear form of the local fractional transport equation was discussed in [37].

\section{Local fractional Burgers' equation}

If the fractal flow is expressed as

$Q=\frac{\phi^{2}}{2}-k \frac{\partial^{\alpha} \phi}{\partial x^{\alpha}}$,

then, using (7), we arrive to the nonlinear local fractional transport equation with a linear nondifferentiable diffusion term 
$\frac{\partial^{\alpha} \phi}{\partial t^{\alpha}}+\frac{\partial^{\alpha}}{\partial x^{\alpha}}\left(\frac{\phi^{2}}{2}-\kappa \frac{\partial^{\alpha} \phi}{\partial x^{\alpha}}\right)=0$,

where $\kappa$ is a diffusion coefficient and $\phi(x, t)$ is a nondifferentiable function.

Equation (12) can be expressed as

$\frac{\partial^{\alpha} \phi}{\partial t^{\alpha}}+\phi \frac{\partial^{\alpha} \phi}{\partial x^{\alpha}}=\kappa \frac{\partial^{2 \alpha} \phi}{\partial x^{2 \alpha}}$.

Equation (13) is the LFBE, and the diffusion coefficient $\kappa$ denotes the fluid kinematic viscosity.

If the quadratic term in (13) is neglected, then we obtain the local fractional diffusion equation (i.e. the LFBE) [35]:

$\frac{\partial^{\alpha} \phi}{\partial t^{\alpha}}-\kappa \frac{\partial^{2 \alpha} \phi}{\partial x^{2 \alpha}}=0$.

From (13), the local fractional forced BE, involving an external force $g_{1}(x, t)$, can be obtained in the form

$\frac{\partial^{\alpha} \phi}{\partial t^{\alpha}}+\phi \frac{\partial^{\alpha} \phi}{\partial x^{\alpha}}=\kappa \frac{\partial^{2 \alpha} \phi}{\partial x^{2 \alpha}}+g_{1}$.

Using (15), the nonlinear local fractional transport equation with a source term $g_{2}(x, t)$ is

$\frac{\partial^{\alpha} \phi}{\partial t^{\alpha}}+\phi \frac{\partial^{\alpha} \phi}{\partial x^{\alpha}}=g_{2}$

In (16), the function $g_{2}(x, t)$ is a non-differentiable source term.

\section{Local fractional derivative transformations}

The local fractional derivative transformation can be used to convert the LFBE into a linear local fractional diffusion equation. The main idea and the transformation approach are explained in the sequel.

Let us define

$\phi=\frac{\partial^{\alpha} \varphi}{\partial x^{\alpha}}$

and

$\frac{\partial^{\alpha} \varphi}{\partial t^{\alpha}}=\kappa \frac{\partial^{\alpha} \phi}{\partial x^{\alpha}}-\frac{\phi^{2}}{2}$.

Then, from expressions (17) and (18), we obtain

$\frac{\partial^{\alpha} \varphi}{\partial t^{\alpha}}=\kappa \frac{\partial^{2 \alpha} \varphi}{\partial x^{2 \alpha}}-\frac{1}{2}\left(\frac{\partial^{\alpha} \varphi}{\partial x^{\alpha}}\right)^{2}$.
Let us define the function

$\psi(x, t)=\sum_{i=0}^{\infty}\left(-\frac{1}{2 \kappa}\right)^{i} \frac{\varphi^{i}(x, t)}{\Gamma(1+i \alpha)}$.

This allows developing the following equations

$$
\begin{aligned}
& \frac{\partial^{\alpha} \psi(x, t)}{\partial t^{\alpha}} \\
& =\left(-\frac{1}{2 \kappa}\right) \frac{\partial^{\alpha} \varphi(x, t)}{\partial t^{\alpha}} \sum_{i=0}^{\infty}\left(-\frac{1}{2 \kappa}\right)^{i} \frac{\varphi^{i}(x, t)}{\Gamma(1+i \alpha)} \\
& =-\frac{1}{2 \kappa} \frac{\partial^{\alpha} \varphi(x, t)}{\partial t^{\alpha}} \psi(x, t),
\end{aligned}
$$

and

$$
\begin{aligned}
& \frac{\partial^{\alpha} \psi(x, t)}{\partial x^{\alpha}} \\
& =\left(-\frac{1}{2 \kappa}\right) \frac{\partial^{\alpha} \varphi(x, t)}{\partial x^{\alpha}} \sum_{i=1}^{\infty}\left(-\frac{1}{2 \kappa}\right)^{i} \frac{\varphi^{i}(x, t)}{\Gamma(1+i \alpha)} \\
& =-\frac{1}{2 \kappa} \frac{\partial^{\alpha} \varphi(x, t)}{\partial x^{\alpha}} \psi(x, t) .
\end{aligned}
$$

In view of (21) and (22), we obtain the local fractional derivative transformation, which is given as follows:

$$
\begin{aligned}
& \phi=\frac{\partial^{\alpha} \varphi(x, t)}{\partial t^{\alpha}}=-2 \kappa \frac{\frac{\partial^{\alpha} \psi(x, t)}{\partial t^{\alpha}}}{\psi(x, t)} \\
& \frac{\partial^{\alpha} \varphi(x, t)}{\partial x^{\alpha}}=-2 \kappa \frac{\frac{\partial^{\alpha} \psi(x, t)}{\partial x^{\alpha}}}{\psi(x, t)}, \\
& \frac{\partial^{2 \alpha} \varphi(x, t)}{\partial x^{2 \alpha}}=2 \kappa\left(\frac{\frac{\partial^{\alpha} \psi(x, t)}{\partial x^{\alpha}}}{\psi(x, t)}\right)^{2}-\frac{2 \kappa}{\psi(x, t)} \frac{\partial^{2 \alpha} \psi(x, t)}{\partial x^{2 \alpha}} .
\end{aligned}
$$

From Eqs. (23), (24) and (25), we convert eq. (19) into

$$
\begin{aligned}
& -2 \kappa \frac{\frac{\partial^{\alpha} \psi(x, t)}{\partial t^{\alpha}}}{\psi(x, t)} \\
& =\kappa\left(2 \kappa\left(\frac{\frac{\partial^{\alpha} \psi(x, t)}{\partial x^{\alpha}}}{\psi(x, t)}\right)^{2}-\frac{2 \kappa}{\psi(x, t)} \frac{\partial^{2 \alpha} \psi(x, t)}{\partial x^{2 \alpha}}\right) \\
& -2 \kappa^{2}\left(\frac{\frac{\partial^{\alpha} \psi(x, t)}{\partial x^{\alpha}}}{\psi(x, t)}\right)^{2} .
\end{aligned}
$$

Finally, the linear local fractional diffusion equation reads as

$$
\frac{\partial^{\alpha} \psi(x, t)}{\partial t^{\alpha}}=\kappa \frac{\partial^{2 \alpha} \psi(x, t)}{\partial x^{2 \alpha}} .
$$


More details about linear local fractional diffusion equation are available in [35].

\section{Discussion}

If the fractal dimension varies from $\alpha$ to 1 , then the conserved density changes into a differentiable conserved density. In this context, we transform (13) into the classical BE [2,3]:

$\frac{\partial \phi}{\partial t}+\phi \frac{\partial \phi}{\partial x}=\kappa \frac{\partial^{2} \phi}{\partial x^{2}}$,

where $\phi(x, t)$ is a differentiable function.

For $\alpha=1$, we have

$\frac{\partial \varphi}{\partial t}=\kappa \frac{\partial^{2} \varphi}{\partial x^{2}}-\frac{1}{2}\left(\frac{\partial \varphi}{\partial x}\right)^{2}$.

With the Cole-Hopf transformation [3-5], we have

$\phi=\frac{\partial \varphi(x, t)}{\partial t}=-2 \kappa \frac{\frac{\partial \psi(x, t)}{\partial t}}{\psi(x, t)}$,

$\frac{\partial \varphi(x, t)}{\partial x}=-2 \kappa \frac{\frac{\partial \psi(x, t)}{\partial x}}{\psi(x, t)}$,

$\frac{\partial^{2} \varphi(x, t)}{\partial x^{2}}=2 \kappa\left(\frac{\frac{\partial \psi(x, t)}{\partial x}}{\psi(x, t)}\right)^{2}-\frac{2 \kappa}{\psi(x, t)} \frac{\partial^{2} \psi(x, t)}{\partial x^{2}}$.

In (20), we suggested a transformation function that in view the case at issue can be written as

$\psi(x, t)=\sum_{i=0}^{\infty}\left(-\frac{1}{2 \kappa}\right)^{i} \frac{\varphi^{i}(x, t)}{\Gamma(1+i)}$.

This transformation function was developed in [5] as

$\psi(x, t)=e^{-\frac{1}{2 \kappa} \varphi(x, t)}$,

which leads to

$\varphi(x, t)=-2 \kappa \ln \psi(x, t)$.

Hence, from (33) and (34), we obtain

$e^{-\frac{1}{2 \kappa} \varphi(x, t)}=\sum_{i=0}^{\infty}\left(-\frac{1}{2 \kappa}\right)^{i} \frac{\varphi^{i}(x, t)}{\Gamma(1+i)}$.

In fact, expression (36) is the Taylor expansion of $e^{-\frac{1}{2 \kappa} \varphi(x, t)}$.

Moreover, using (20), we may develop the local fractional series expansion of $E_{\alpha}(-\varphi(x, t) / 2 \kappa)$ with a non-differentiable function $\varphi(x, t)$, namely
$E_{\alpha}\left(-\frac{1}{2 \kappa} \varphi(x, t)\right)=\sum_{i=0}^{\infty}\left(-\frac{1}{2 \kappa}\right)^{i} \frac{\varphi^{i}(x, t)}{\Gamma(1+i \alpha)}$.

\section{Conclusions}

The communication discussed the LFBE which can be developed on the basis of the nonlinear local fractional transport equation with a linear non-differentiable diffusion term. Consequently, the local fractional derivative transformation conceived by the presented analysis allowed transforming the LFBE into the local fractional diffusion equation. The classical $\mathrm{BE}$ emerges as a reasonable consequence from the LFBE when the fractal dimension $\alpha$ becomes equal to 1 .

\section{References}

1. Burgers, J.M.: A mathematical model illustrating the theory of turbulence. Adv. Appl. Mech. 1, 171-199 (1948)

2. Burgers, J.M.: The Nonlinear Diffusion Equation. Springer, Berlin (1974)

3. Whitham, G.B.: Linear and Nonlinear Waves. Wiley, New York (2011)

4. Cole, J.D.: On a quasi-linear parabolic equation occurring in aerodynamics. Quart. Appl. Math. 9(3), 225-236 (1951)

5. Hopf, E.: The partial differential equation $u t+u u x=u x x$. Commun. Pure Appl. Math. 3, 201-230 (1950)

6. Lax, P.D.: Hyperbolic systems of conservation laws II. Commun. Pure Appl. Math. 10, 537-566 (1957)

7. Avrin, J.D.: The generalized Burgers' equation and the Navier-Stokes equation in Rn with singular initial data. Proc. Am. Math. Soc. 101(1), 29-40 (1987)

8. Hamanaka, M., Toda, K.: Noncommutative Burgers equation. J. Phys. A 36(48), 11981 (2003)

9. Haselwandter, C., Vvedensky, D.D.: Fluctuations in the lattice gas for Burgers' equation. J. Phys. A 35(41), L579 (2002)

10. Da Prato, G., Debussche, A., Temam, R.: Stochastic Burgers' equation. NoDEA 1(4), 389-402 (1994)

11. Gurbatov, S.N., Simdyankin, S.I., Aurell, E., Frisch, U., Toth, G.: On the decay of Burgers turbulence. J. Fluid Mech. 344, 339-374 (1997)

12. Musha, T., Higuchi, H.: Traffic current fluctuation and the Burgers equation. Jpn. J. Appl. Phys. 17(5), 811-816 (1978)

13. Blackstock, D.T.: Generalized Burgers equation for plane waves. J. Acoustical. Soc. Am. 77(6), 2050-2053 (1985)

14. Caldwell, J., Wanless, P., Cook, A.E.: A finite element approach to Burgers' equation. Appl. Math. Mod. 5(3), 189193 (1981)

15. Kakuda, K., Tosaka, N.: The generalized boundary element approach to Burgers' equation. Int. J. Numer. Meth. Eng. 29(2), 245-261 (1990)

16. Wazwaz, A.M.: Multiple-front solutions for the Burgers equation and the coupled Burgers equations. Appl. Math. Comput. 190(2), 1198-1206 (2007) 
17. Kilbas, A.A.A., Srivastava, H.M., Trujillo, J.J.: Theory and Applications of Fractional Differential Equations. Elsevier, Amsterdam (2006)

18. Podlubny, I.: Fractional Differential Equations. Academic Press, Waltham (1999)

19. West, B., Bologna, M., Grigolini, P.: Physics of Fractal Operators. Springer, Berlin (2003)

20. Baleanu, D., Diethelm, K., Scalas, E., Trujillo, J.J.: Fractional Calculus Models and Numerical Methods, Series on Complexity, Nonlinearity and Chaos. World Scientific, Singapore (2012)

21. Atangana, A.: Convergence and stability analysis of a novel iteration method for fractional biological population equation. Neural Comput. Appl. 25(5), 1021-1030 (2014)

22. Biler, P., Funaki, T., Woyczynski, W.A.: Fractal Burgers equations. J. Differ. Equ. 148(1), 9-46 (1998)

23. Alibaud, N., Imbert, C., Karch, G.: Asymptotic properties of entropy solutions to fractal Burgers equation. SIAM J. Math. Anal. 42(1), 354-376 (2010)

24. Karch, G., Miao, C., Xu, X.: On convergence of solutions of fractal Burgers equation toward rarefaction waves. SIAM J. Math. Anal. 39(5), 1536-1549 (2008)

25. Sugimoto, N.: Burgers equation with a fractional derivative; hereditary effects on nonlinear acoustic waves. J. Fluid Mech. 225, 631-653 (1991)

26. Chan, C.H., Czubak, M., Silvestre, L.: Eventual regularization of the slightly supercritical fractional Burgers equation. Discrete Contin. Dyn. Syst. 27(2), 847-861 (2010)

27. Yildırım, A., Mohyud-Din, S.T.: Analytical approach to space-and time-fractional burgers equations. Chin. Phys. Letts. 27(9), 090501 (2010)

28. Momani, S.: Non-perturbative analytical solutions of the space-and time-fractional Burgers equations. Chaos Solitons Fractals 28(4), 930-937 (2006)

29. Xu, Y., Agrawal, O.P.: Numerical solutions and analysis of diffusion for new generalized fractional Burgers equation. Fract. Calc. Appl. Anal. 16(3), 709-736 (2013)

30. Wu, G.C., Baleanu, D.: Variational iteration method for the Burgers' flow with fractional derivatives-new Lagrange multipliers. Appl. Math. Mod. 37(9), 6183- 6190 (2013)
31. Chen, Y., An, H.L.: Numerical solutions of coupled Burgers equations with time-and space-fractional derivatives. Appl. Math. Comput. 200(1), 87-95 (2008)

32. Khan, N.A., Ara, A., Mahmood, A.: Numerical solutions of time-fractional Burgers equations: a comparison between generalized differential transformation technique and homotopy perturbation method. Inter. J. Numer. Meth. Heat Fluid Flow 22(2), 175-193 (2012)

33. Yang, X.J.: Advanced Local Fractional Calculus and Its Applications. World Science, New York (2012)

34. Yang, X.J., Srivastava, H.M., He, J.H., Baleanu, D.: Cantortype cylindrical-coordinate method for differential equations with local fractional derivatives. Phys. Letts. A 377(28), 1696-1700 (2013)

35. Yang, X.J., Baleanu, D., Zhong, W.P.: Approximate solutions for diffusion equations on cantor space-time. Proc. Rom. Acad. Ser. A 14(2), 127-133 (2013)

36. Zhang, Y., Baleanu, D., Yang, X.J.: On a local fractional wave equation under fixed entropy arising in fractal hydrodynamics. Entropy 16(12), 6254-6262 (2014)

37. Wang, L. F., Yang, X. J., Baleanu, D., Cattani, C., Zhao, Y.: Fractal dynamical model of vehicular traffic flow within the local fractional conservation laws. Abstr. Appl. Anal. 2014(635760), 5 (2014)

38. Yang, X.J., Baleanu, D., Machado, J.A.T.: Mathematical aspects of the Heisenberg uncertainty principle within local fractional Fourier analysis. Bound Value Probl. 2013(1), 1$16(2013)$

39. Liu, H.Y., He, J.H., Li, Z.B.: Fractional calculus for nanoscale flow and heat transfer. Inter. J. Numer. Meth. Heat Fluid Flow 24(6), 1227-1250 (2014)

40. Kolwankar, K.M., Gangal, A.D.: Hölder exponents of irregular signals and local fractional derivatives. Pramana 48(1), 49-68 (1997)

41. Babakhani, A., Daftardar-Gejji, V.: On calculus of local fractional derivatives. J. Math. Anal. Appl. 270(1), 66-79(2002)

42. Carpinteri, A., Chiaia, B., Cornetti, P.: The elastic problem for fractal media: basic theory and finite element formulation. Comput. Struct. 82(6), 499-508 (2004) 